This is an electronic reprint of the original article. This reprint may differ from the original in pagination and typographic detail.

\author{
Author(s): Skalyga, V.; Izotov, I.; Golubev, S.; Sidorov, A.; Razin, S.; Vodopyanov, A.; Tarvainen, \\ Olli; Koivisto, Hannu; Kalvas, Taneli
}

Title: $\quad$ New progress of high current gasdynamic ion source (invited)

Year: $\quad 2016$

Version:

Please cite the original version:

Skalyga, V., Izotov, I., Golubev, S., Sidorov, A., Razin, S., Vodopyanov, A., Tarvainen, O., Koivisto, H., \& Kalvas, T. (2016). New progress of high current gasdynamic ion source (invited). Review of Scientific Instruments, 87(2), Article 02A716. https://doi.org/10.1063/1.4934213

All material supplied via JYX is protected by copyright and other intellectual property rights, and duplication or sale of all or part of any of the repository collections is not permitted, except that material may be duplicated by you for your research use or educational purposes in electronic or print form. You must obtain permission for any other use. Electronic or print copies may not be offered, whether for sale or otherwise to anyone who is not an authorised user. 


\section{AIP | Review of Scientific Instruments}

\section{New progress of high current gasdynamic ion source (invited)}

V. Skalyga, I. Izotov, S. Golubev, A. Sidorov, S. Razin, A. Vodopyanov, O. Tarvainen, H. Koivisto, and T. Kalvas

Citation: Review of Scientific Instruments 87, 02 A716 (2016); doi: 10.1063/1.4934213

View online: http://dx.doi.org/10.1063/1.4934213

View Table of Contents: http://scitation.aip.org/content/aip/journal/rsi/87/2?ver=pdfcov

Published by the AIP Publishing

\section{Articles you may be interested in}

Extracted ion current density in close-coupling multi-antenna type radio frequency driven ion source: CCMATISa)

Rev. Sci. Instrum. 85, 02B310 (2014); 10.1063/1.4830363

Langmuir probe diagnostics of plasma in high current electron cyclotron resonance proton ion source

Rev. Sci. Instrum. 84, 073303 (2013); 10.1063/1.4813279

Fourth generation electron cyclotron resonance ion sources (invited)a)

Rev. Sci. Instrum. 79, 02A321 (2008); 10.1063/1.2816793

New developments in multicusp $\mathrm{H}$ - ion sources for high energy accelerators (invited)a)

Rev. Sci. Instrum. 79, 02A515 (2008); 10.1063/1.2801647

High current density production of multicharged ions with ECR plasma heated by gyrotron transmitter Rev. Sci. Instrum. 73, 528 (2002); 10.1063/1.1430030

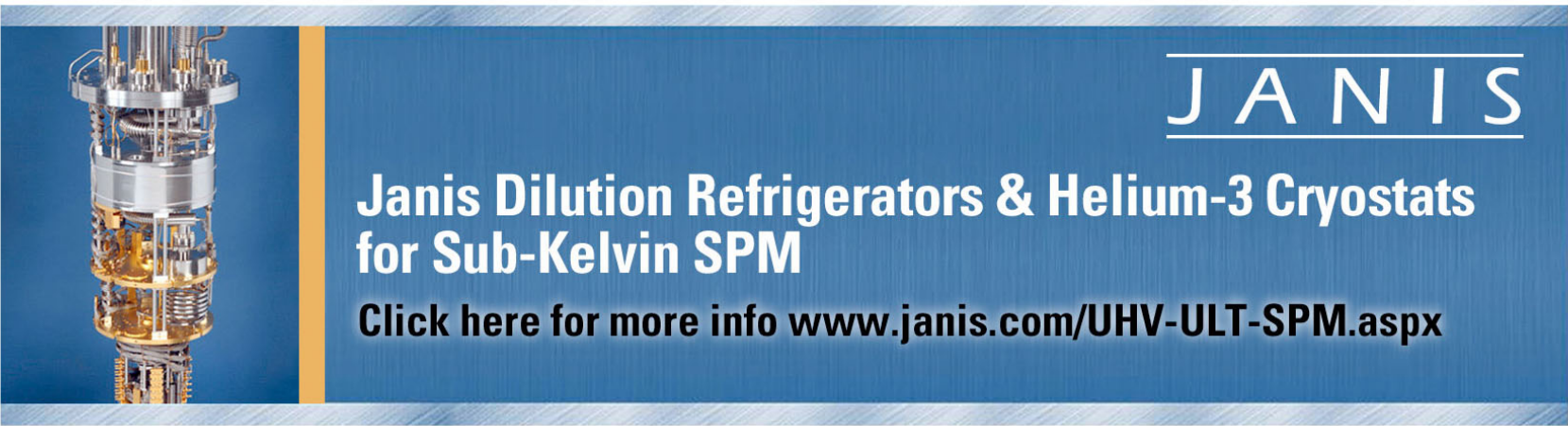




\title{
New progress of high current gasdynamic ion source (invited)
}

\author{
V. Skalyga, ${ }^{1,2, a)}$ I. Izotov, ${ }^{1}$ S. Golubev, ${ }^{1}$ A. Sidorov, ${ }^{1,2}$ S. Razin, ${ }^{1}$ A. Vodopyanov, ${ }^{1,2}$ \\ O. Tarvainen, ${ }^{3}$ H. Koivisto ${ }^{3}$ and T. Kalvas ${ }^{3}$ \\ ${ }^{1}$ Institute of Applied Physics, Russian Academy of Sciences (IAP RAS), 46 Ul'yanova St., \\ 603950 Nizhny Novgorod, Russia \\ ${ }^{2}$ Lobachevsky State University of Nizhny Novgorod (UNN), 23 Gagarina St., 603950 Nizhny Novgorod, Russia \\ ${ }^{3}$ Department of Physics, University of Jyvaskyla, P.O. Box 35 (YFL), 40500 Jyvaskyla, Finland
}

(Presented 27 August 2015; received 5 September 2015; accepted 8 October 2015; published online 26 October 2015)

\begin{abstract}
The experimental and theoretical research carried out at the Institute of Applied Physics resulted in development of a new type of electron cyclotron resonance ion sources (ECRISs) - the gasdynamic ECRIS. The gasdynamic ECRIS features a confinement mechanism in a magnetic trap that is different from Geller's ECRIS confinement, i.e., the quasi-gasdynamic one similar to that in fusion mirror traps. Experimental studies of gasdynamic ECRIS were performed at Simple Mirror Ion Source (SMIS) 37 facility. The plasma was created by 37.5 and $75 \mathrm{GHz}$ gyrotron radiation with power up to $100 \mathrm{~kW}$. High frequency microwaves allowed to create and sustain plasma with significant density (up to $8 \times 10^{13} \mathrm{~cm}^{-3}$ ) and to maintain the main advantages of conventional ECRIS such as high ionization degree and low ion energy. Reaching such high plasma density relies on the fact that the critical density grows with the microwave frequency squared. High microwave power provided the average electron energy on a level of $50-300 \mathrm{eV}$ enough for efficient ionization even at neutral gas pressure range of $10^{-4}-10^{-3}$ mbar. Gasdynamic ECRIS has demonstrated a good performance producing high current (100-300 mA) multi-charged ion beams with moderate average charge ( $Z=4-5$ for argon). Gasdynamic ECRIS has appeared to be especially effective in low emittance hydrogen and deuterium beams formation. Proton beams with current up to $500 \mathrm{emA}$ and RMS emittance below $0.07 \pi \cdot \mathrm{mm} \cdot \mathrm{mrad}$ have been demonstrated in recent experiments. (C) 2015 AIP Publishing LLC. [http://dx.doi.org/10.1063/1.4934213]
\end{abstract}

\section{INTRODUCTION}

Investigations of pulsed electron cyclotron resonance (ECR) discharge in an open magnetic trap under conditions of powerful ECR heating with gyrotron $\mathrm{mm}$-waveband radiation were carried out over the last 20 years at the Institute of Applied Physics (IAP RAS, Nizhniy Novgorod, Russia) ${ }^{1-5}$ In the beginning, the work was devoted to development of a high frequency ECR source of multi-charged ions with outstanding parameters of plasma heating $(37.5 \mathrm{GHz}, 100 \mathrm{~kW})$. According to Geller's scaling laws, ${ }^{6}$ such increase in frequency and power in comparison to conventional electron cyclotron resonance ion source (ECRIS) was expected to boost the ion source performance and provide a significant progress in ECRIS development. However, due to short pulse operation mode and low repetition rate of the used gyrotrons (pulse duration $<1 \mathrm{~ms}, 0.1 \mathrm{~Hz}$ ), breakdown and discharge conditions similar to a conventional ECRIS were unreachable. The minimum neutral gas pressure was two orders higher $\left(10^{-4} \mathrm{mbar}\right)$ and the plasma parameters differed significantly from conventional ECRIS. After years, this work resulted in development of a new type of ion source-high current gasdynamic ion source.

\footnotetext{
Note: Invited paper, published as part of the Proceedings of the 16th International Conference on Ion Sources, New York, New York, USA, August 2015 .

a) Author to whom correspondence should be addressed. Electronic mail: skalyga@ipfran.ru
}

\section{QUASI-GASDYNAMIC PLASMA CONFINEMENT}

The use of powerful mm-band radiation allows to increase the plasma density in the discharge significantly (proportional to the square of the radiation frequency ${ }^{4-7}$ ) in comparison to conventional ECRISs, which utilize microwave radiation with frequencies on the order of $10 \mathrm{GHz} .{ }^{6}$ In experiments with $37.5 \mathrm{GHz}$ and $75 \mathrm{GHz}$ gyrotrons, the plasma density reaches values of $10^{13}-10^{14} \mathrm{~cm}^{-3} .{ }^{8,9}$ Significant increase of the plasma density leads to a change of the confinement mode. A so-called quasi-gasdynamic confinement ${ }^{4,5}$ was realized in the presented experiments instead of the collision-less confinement, ${ }^{10}$ which is typical for modern ECRISs. The transition from collisionless to quasi-gasdynamic confinement occurs when the plasma density is high enough for the scattering rate of electrons into the loss-cone to be higher than the maximum possible electron loss rate caused by the ion-sound flux through the magnetic mirrors. ${ }^{11}$ In such situation, the loss-cone in the velocity space is populated, and the plasma lifetime does not depend on the collisional electron scattering rate into the losscone, i.e., on the plasma density, but is determined by the trap size, magnetic field structure, and ion sound velocity. ${ }^{11}$ The plasma lifetime, which is much shorter than in conventional classical ECRISs, can be expressed as $\tau=(\mathrm{L} \times \mathrm{LR}) /(2 \mathrm{Vis})$, where $\mathrm{L}$ is the magnetic trap length, $\mathrm{R}$ is the trap mirror ratio (ratio between magnetic field in the magnetic mirror and in the trap center), and Vis is the ion sound velocity. Short plasma lifetime provides high plasma flux density from the trap. The flux is proportional to the plasma density and ion lifetime, i.e., 
$\mathrm{I} \sim \mathrm{N} / \tau$, where $\mathrm{N}$ is the plasma density. Due to the high plasma density, the confinement parameter $\mathrm{Ne} \cdot \tau$, which determines the ionization degree and average ion charge, can be as high as $10^{8}-10^{9} \mathrm{~s} \cdot \mathrm{cm}^{-3}$, which is enough for efficient ionization. The main advantages of quasi-gasdynamic confinement are the following. The plasma lifetime does not depend on its density, and therefore, increase of the density would lead to rising of confinement parameter and average ion charge. In addition, the plasma lifetime is proportional to the magnetic trap length and the source performance could be improved by adjusting the trap length. ECR sources running under conditions of such plasma confinement are called gasdynamic ECRISs.

Possibilities of the gasdynamic confinement are demonstrated in this paper with experimental results obtained at Simple Mirror Ion Source (SMIS) 37 facility. ${ }^{4,5}$ Investigation results on multi-charged ions production under condition of powerful gyrotron plasma heating with 37.5 and $75 \mathrm{GHz}$ are presented. It is shown that the described peculiarities of quasigasdynamic ECR discharge sustained by mm-waveband radiation, namely, short lifetime and high density, provide unprecedented ion current densities up to $800 \mathrm{emA} / \mathrm{cm}^{2}$.

\section{SMIS 37 EXPERIMENTAL FACILITY}

The main part of the experiments was conducted at SMIS 37 facility. During the years, its configuration has been changing slightly, the latest one being schematically depicted in Fig. 1 . The plasma was created and sustained inside a $\mathrm{d}=4 \mathrm{~cm}$ vacuum chamber (placed in a magnetic trap) by pulsed (1 ms) $37.5 \mathrm{GHz}$ or $75 \mathrm{GHz}$ linearly polarized gyrotron radiation with power up to $100 \mathrm{~kW}$. The simple mirror magnetic field was created by means of pulsed solenoids positioned at a distance of $15 \mathrm{~cm}$ from each other, providing a mirror ratio of 5 . The magnetic field strength was varied in a range of 1-4 $\mathrm{T}$ at mirror plugs, whereas the resonant field strength is $1.34 \mathrm{~T}$ for $37.5 \mathrm{GHz}$ and $2.7 \mathrm{~T}$ for $75 \mathrm{GHz}$. The microwave radiation was coupled to the chamber quasi-optically through a quartz window and a special coupling system, which protects the window from the plasma flux. The pulsed gas feed line was incorporated into the coupling system, i.e., the neutral gas was injected axially.

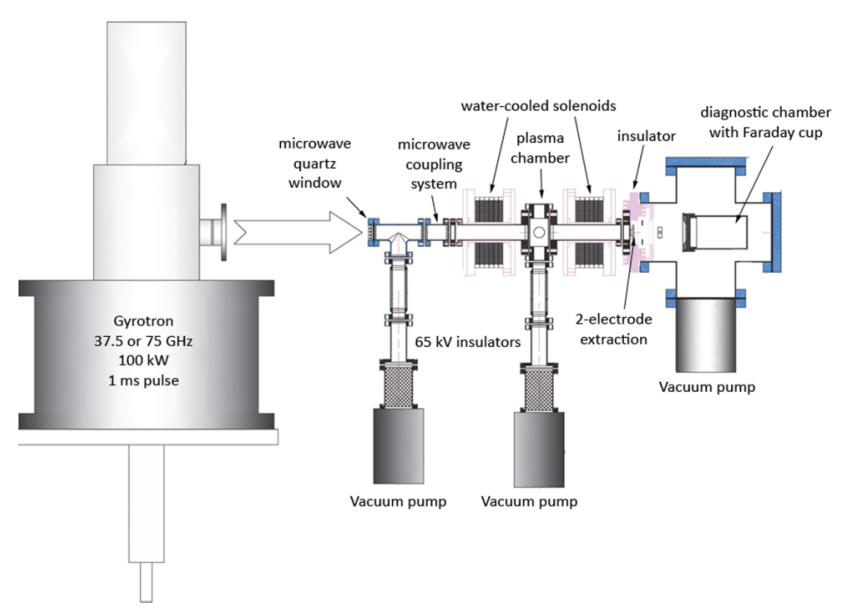

FIG. 1. Schematic view of SMIS 37 experimental facility.

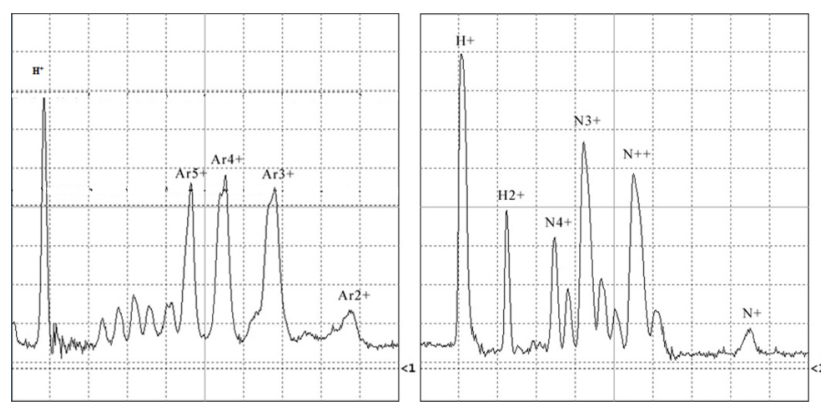

FIG. 2. Argon and nitrogen spectra. ECR plasma heating with $37.5 \mathrm{GHz}$, $100 \mathrm{~kW}$ gyrotron radiation in a simple mirror trap.

The ion extraction and beam formation were realized by a two-electrode (diode) system consisting of a plasma electrode and a puller. The diameter of the extraction aperture was varied from 1 to $10 \mathrm{~mm}$. The distance between the extraction system and the magnetic plug at the center of the solenoid magnet was designed to be variable, which allows tuning the plasma flux density at the plasma electrode. The maximum applied extraction voltage was $60 \mathrm{kV}$, which was an absolute maximum for the used power supply. A Faraday cup with an aperture of $85 \mathrm{~mm}$ was placed right behind the puller (grounded hollow electrode) to capture the whole beam. The cup was equipped with an electrostatic secondary electron suppression. A $42^{\circ}$ bending magnet was installed downstream in the beam line for measuring extracted beam spectrum.

\section{MULTICHARGED IONS PRODUCTION}

A number of papers were devoted to multi-charged beam production at SMIS 37. ${ }^{1-5}$ In this paper, the main results obtained some years ago are shown to demonstrate the typical source performance. In Fig. 2, two ion spectra with nitrogen and argon are presented in the case of $37.5 \mathrm{GHz}, 100 \mathrm{~kW}$ plasma heating.

The effect of plasma density increase within gasdynamic confinement with increase of microwave frequency is shown in Fig. 3. Helium ion spectra for 37.5 and $75 \mathrm{GHz}, 100 \mathrm{~kW}$ heating demonstrate a great improvement in average ion charge.

In these experiments, a single aperture two electrode extraction system with $1 \mathrm{~mm}$ hole was used for beam formation providing total ion current up to $5 \mathrm{~mA} .^{2}$ The experiments were repeated later with multi-aperture extraction systems. Extracted ion current dependence on the accelerating voltage in case of 13-hole (each $3 \mathrm{~mm}$ in diameter) plasma electrode is shown in Fig. 4.

Presented results demonstrate that gasdynamic ion source is able to produce hundreds of emA of moderately charged
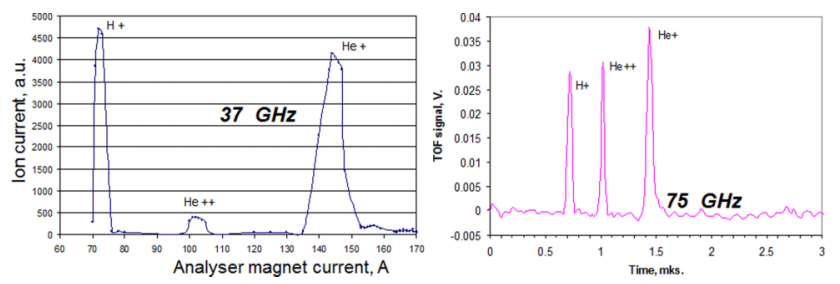

FIG. 3. Helium spectra. 37.5, $100 \mathrm{~kW}$ ECR heating (left) and $75 \mathrm{GHz}$, $100 \mathrm{~kW}$ ECR heating (right). 

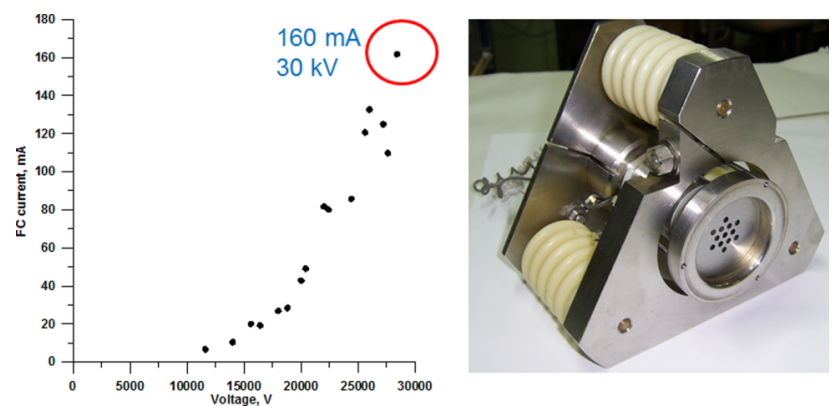

FIG. 4. Faraday cup current dependence on extraction voltage (left) obtained with multi-aperture extraction system (right).

( $Q$ up to 6+) beams. Low emittance and high current of such beams may allow using them together with charge-breeding or stripping techniques. Further increase of the microwave frequency is promising for the production of high current heavy ion beams with the average charge about +10 and their injection into accelerators with strippers after first acceleration stage.

\section{PROTON AND DEUTERON BEAMS FORMATION}

Operation of modern high power accelerators often requires production of intense proton and deuterium beams. $\mathrm{H}+$ beams are utilized or envisioned for use in linear accelerators, e.g., the future European Spallation Source under design; ${ }^{12,13}$ some special applications such as neutron generators or the International Fusion Materials Irradiation Facility (IFMIF) project, ${ }^{3}$ require D+ (deuteron) ion beams. Requirements for the brightness of such beams grow together with the demand of accelerator development and arising experimental needs. New facilities aiming at outperforming the previous generation accelerators are usually designed for higher beam currents. Enhancing the beam intensity and maintaining low transverse emittance at the same time are, however, quite challenging tasks. The most modern accelerators require $\mathrm{H}+/ \mathrm{D}+$ ion beams with currents up to hundreds of emA (pulsed or CW) and normalized emittance less than $0.2 \pi \cdot \mathrm{mm} \cdot \mathrm{mrad}^{12,14}$ to keep the beam losses at high energy sections of the linacs below commonly imposed $1 \mathrm{~W} / \mathrm{m}$ limit. Previous experiments on heavy multi-charged ion production demonstrated that gasdynamic ion source is able to produce ion beams with record beam current density and moderate ion charge. The average electron energy in plasma of ECR discharge with quasi-gasdynamic confinement sustained by gyrotron radiation varies from 50 to $300 \mathrm{eV}$ and it is optimal for efficient hydrogen ionization. Due to this coincidence, it was decided to test the gasdynamic ECR source performance for proton and deuteron beams formation. In previous papers, ${ }^{15,16}$ it was demonstrated that proton beams with current of hundreds of $\mathrm{mA}$ could be produced. The latest results are presented below.

A single-aperture extraction system was used for beam formation in the presented experiments. As only two fixed puller holes were available (i.e., 10 and $22 \mathrm{~mm}$ in diameter), the optimization of extraction electrode configuration was done varying the gap between the electrodes. Plasma electrodes with aperture diameters 5 and $10 \mathrm{~mm}$ were used. In the case of $5 \mathrm{~mm}$
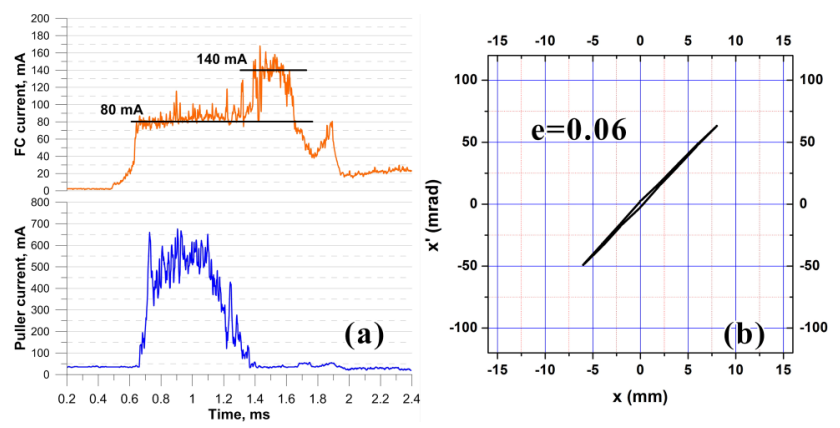

FIG. 5. Hydrogen, $5 \mathrm{~mm}$ plasma electrode hole. (a) Faraday cup and puller currents. (b) RMS emittance diagram.

extraction hole, the optimal gap between electrodes appeared to be $10 \mathrm{~mm}$, yielding the maximum beam current with a puller aperture of $10 \mathrm{~mm}$. The Faraday cup and puller currents are shown in Fig. 5(a), total beam current grows from $80 \mathrm{~mA}$ at steady state up to $140 \mathrm{~mA}$ at the end of the microwave pulse, which is explained by decrease of the plasma density towards the optimum value for used configuration and accelerating voltage of $42 \mathrm{kV}$ and subsequently improved beam transport. Transversal emittance (hereinafter normalized RMS) diagram is presented in Fig. 5(b), showing value of $0.06 \pi \mathrm{mm}$ mrad.

The biggest hole diameter in plasma electrode was $10 \mathrm{~mm}$. In this case, the optimal gap between electrodes for $10 \mathrm{~mm}$ plasma electrode aperture appeared to be $6 \mathrm{~mm}$, while the puller hole diameter was $22 \mathrm{~mm}$. The Faraday cup and puller currents are shown in Fig. 6(a). The total beam current remains relatively stable at the level of $450 \mathrm{~mA}$ through $70 \%$ of the microwave pulse. Accelerating voltage of $41.5 \mathrm{kV}$ was used. Transversal emittance diagram is presented in Fig. 6(b), showing a RMS value of $0.07 \pi \mathrm{mm}$ mrad.

The experiments with deuterium were performed under similar conditions. The extraction system configuration was the same as the latter for hydrogen $(10 \mathrm{~mm}$ plasma electrode hole, $6 \mathrm{~mm}$ gap, $22 \mathrm{~mm}$ puller electrode hole). Source settings were adjusted slightly from the optimal ones for proton beam to maximize the total current. The Faraday cup current together with puller current is shown in Fig. 7(a). The total beam current rapidly reaches a value of $400 \mathrm{~mA}$, then slowly increases to $500 \mathrm{~mA}$, and remains there till the end of the microwave pulse. Accelerating voltage of $42 \mathrm{kV}$ was used. Transversal emittance diagram is presented in Fig. 7(b), showing RMS value of $0.07 \pi \cdot \mathrm{mm} \cdot \mathrm{mrad}$.
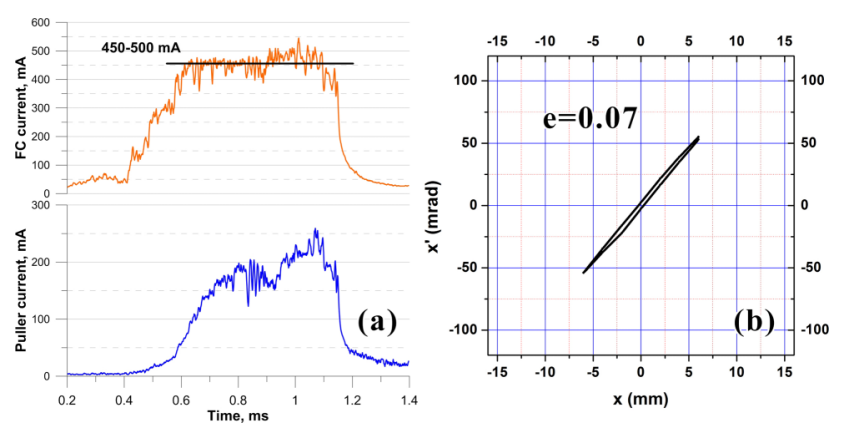

FIG. 6. Hydrogen, $10 \mathrm{~mm}$ plasma electrode hole (a) Faraday cup and puller currents, (b) RMS emittance diagram. 

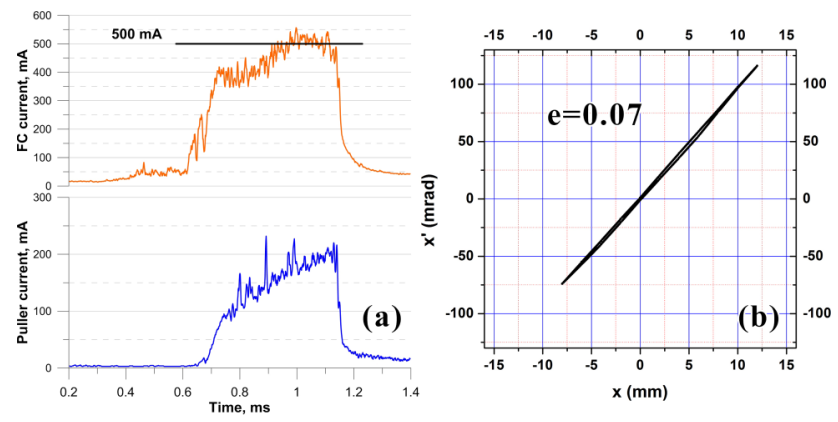

FIG. 7. Deuterium, $10 \mathrm{~mm}$ plasma electrode hole (a) Faraday cup and puller currents, (b) RMS emittance diagram.

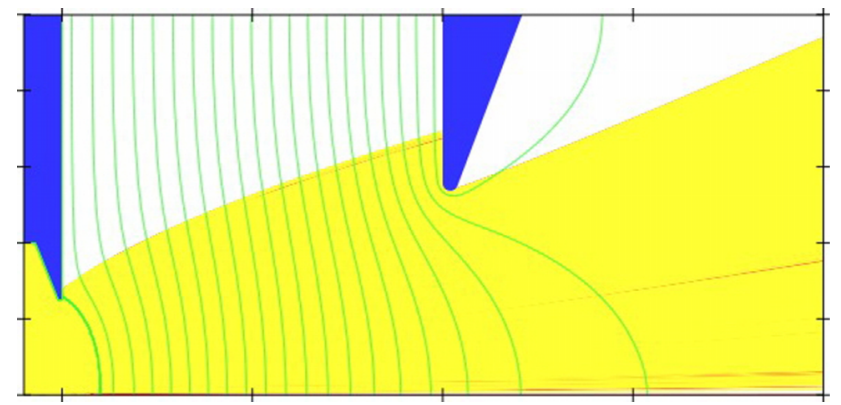

FIG. 8. Ion traces simulated with IBSimu code, $10 \mathrm{~mm}$ case, hydrogen plasma, $45 \mathrm{kV}$.

Figures 5-7 show that measured beam emittance value in contradiction with the theory does not strongly depend on the plasma electrode aperture. In case of presented results, this means that emittance measurement scheme does not have enough precision and calculated values must be considered as the upper limit estimation of the real beam emittance.

The presented results demonstrate the prospects of the high current gasdynamic ECR source for light ion beams production. The maximum RMS brightness of extracted beam reached $100 \mathrm{~A} /(\pi \cdot \mathrm{mm} \cdot \mathrm{mrad})^{2}$. The proton (deuteron) fraction in extracted beams was about $94 \%$ as it was shown in Ref. 15 . It should be added that in presented studies, the total beam current was limited by the accelerating voltage available, which can be seen from Fig. 8, where ion traces are simulated with IBSimu software ${ }^{17}$ for hydrogen plasma, $10 \mathrm{~mm}$ extraction hole diameter and $45 \mathrm{kV}$ accelerating voltage. It is clearly seen that the beam is under-focused due to insufficient electric field.

The extracted beam current could be further enhanced by moving the plasma electrode closer to the magnetic mirror and scaling the extraction voltage and geometry appropriately. According to simulations, the extracted current may eventually exceed $1 \mathrm{~A}$ while maintaining the low emittance. Such result would outperform the conventional ECRISs by a great margin.

\section{CONCLUSION}

The presented results demonstrate the main prospects of the gasdynamic ECRIS. This type of ECRIS has already demonstrated its benefits for light ion beam production. Further studies could significantly increase its performance in multi-charged beam formation. One of the most promising new ion sources which may demonstrate all capabilities of gasdynamic confinement is the Sixty GHz ECR Ion Source using Megawatt Magnets (SEISM), $60 \mathrm{GHz}$ ECRIS at LPSC, Grenoble. ${ }^{18}$ Due to high frequency and power (up to $200 \mathrm{~kW}$ ) of the gyrotron used for plasma creation, it could operate with plasma parameters suitable for gasdynamic confinement with high ionization efficiency. It has been already demonstrated that ion beams with current density up to $800 \mathrm{~mA} / \mathrm{cm}^{2}$ can be produced with this source. The Grenoble facility has a number of advantages in comparison with SMIS 37. The first is a high repetition rate (up to $2 \mathrm{~Hz}$ ) which allows better control of plasma parameters due to satisfactory wall conditioning. The second is the cusp magnetic field of high intensity (up to $8 \mathrm{~T}$ ) and with closed ECR zone. It is the first ion source which can operate effectively in gasdynamic mode having a closed-ECR field, which is of great importance for trapping of energetic electrons. Therefore, interesting results are foreseen from SEISM source, as it may be the first ECRIS able to operate in-between of gasdynamic and traditional collisionless confinement, thus producing high currents and charges.

Another direction of gasdynamic sources evolution is to apply them to $\mathrm{CW}$ operation to produce $\mathrm{CW}$ high current beams for different applications. First results on such studies with $\mathrm{CW}$ discharge sustained with $24 \mathrm{GHz}$ gyrotron are presented in Ref. 19. It was shown that dense ECR discharge can be sustained in $\mathrm{CW}$ mode producing plasma fluxes with $1 \mathrm{eA} / \mathrm{cm}^{2}$ density.

\section{ACKNOWLEDGMENTS}

The Work was supported by RFBR Grant No. 13-0800845 and the grant of the President of Russian Federation for young scientists No. MK-6565.2014.2. Work of Ivan Izotov was supported by the fellowship of the President of Russian Federation for young scientists No. SP-7279.2013.2. The Work was supported by the Academy of Finland under the Finnish Centre of Excellence Programme 2012-2017 (Nuclear and Accelerator Based Physics Research at JYFL) and researcher mobility Grant Nos. 267174 and 267227.

${ }^{1}$ S. V. Golubev et al., Rev. Sci. Instrum. 75(5), 1675 (2004).

${ }^{2}$ S. V. Golubev et al., Trans. Fusion Sci. Technol. 47(1T), 345 (2005).

${ }^{3}$ A. Sidorov et al., Rev. Sci. Instrum. 77(3), 03A341 (2006).

${ }^{4}$ V. Skalyga et al., Plasma Sources Sci. Technol. 15, 727 (2006).

${ }^{5}$ S. Golubev et al., Nucl. Instrum. Methods Phys. Res., Sect. B 256, 537 (2007).

${ }^{6}$ R. Geller, Electron Cyclotron Resonance Ion Sources and ECR Plasmas (Institute of Physics, Bristol, 1996).

${ }^{7}$ M. A. Dorf, Nucl. Instrum. Methods Phys. Res., Sect. A 733, 107 (2014).

${ }^{8}$ S. V. Golubev et al., Rev. Sci. Instrum. 71(2), 669 (2000).

${ }^{9}$ A. V. Vodopyanov et al., High Energy Phys. Nucl. Phys. 31(S1), 152 (2007).

${ }^{10}$ V. Pastukhov, Vopr. Teor. Plasma 13, 160 (1984).

${ }^{11}$ V. V. Mirnov and D. D. Ryutov, Pisma v Zhurnal Theknicheskoi Fiziki 5, 678 (1979).

${ }^{12} \mathrm{~S}$. Gammino et al., in LINAC2010, THP116, Tsukuba, Japan, 12-17 September 2010, http://www.jacow.org, p. 1010.

${ }^{13}$ M. Lindroos et al., Nucl. Instrum. Methods Phys. Res., Sect. B 269, 3258 (2011).

${ }^{14}$ R. Gobin et al., Rev. Sci. Instrum. 83, 02 A345 (2012).

${ }^{15}$ V. Skalyga et al., Rev. Sci. Instrum. 85(2), $02 \mathrm{~A} 702$ (2014).

${ }^{16}$ V. Skalyga et al., J. Instrum. 7, P10010 (2012).

${ }^{17}$ T. Kalvas et al., Rev. Sci. Instrum. 81, $02 B 703$ (2010).

${ }^{18} \mathrm{~T}$. Thuillier et al., in Proceedings of Science (Nufact08) (Scuola Internazionale Superiore di Studi Avanzati, 2008), p. 089.

${ }^{19}$ V. Skalyga et al., "First experiments with gasdynamic ion source in $\mathrm{CW}$ mode," Rev. Sci. Instrum. (these proceedings). 